\title{
CAPÍTULO 17: PRODUÇÃO SUSTENTÁVEL DE ALIMENTOS UTILIZANDO ZEÓLITAS COMO FERTILIZANTES DE LIBERAÇÃO LENTA: UMA REVISÃO
}

\section{CAPÍTULO 17: PRODUCCIÓN DE ALIMENTOS SOSTENIBLE CON ZEOLIT COMO FERTILIZANTES DE LIBERACIÓN LENTA: UNA REVISIÓN}

\section{CHAPTER 17: SUSTAINABLE FOOD PRODUCTION USING ZEOLITE AS SLOW RELEASE FERTILIZERS: A REVIEW}

\begin{abstract}
Lisandro Simão ${ }^{1}$; Carlos Rafael Silva de Oliveira ${ }^{2}$; Luís Antonio Lourenço ${ }^{3}$; Afonso Henrique da Silva Júnior ${ }^{4}$; Éllen Francine Rodrigues ${ }^{5}$
\end{abstract}

DOI: https://doi.org/10.31692/978-65-88970-19-5.242-264

\section{RESUMO}

Os fertilizantes são intencionalmente adicionados no solo ou na superfície da planta com o objetivo de fornecer os nutrientes necessários durante sua vida e assim aumentar a produtividade. Os principais nutrientes utilizados na agricultura são nitrogênio, fósforo e potássio, sendo o conjunto destes macronutrientes chamados de N-P-K. O uso indiscriminado e de longo prazo de fertilizantes tem se tornado uma fonte relevante de poluição do solo, ar e água. O desequilíbrio entre as taxas de liberação dos nutrientes oriundos dos fertilizantes e as taxas de absorção pelas plantas, tornam o processo de fertilização do solo menos eficientes. Para resolver este problema, pode-se usar fertilizantes que tem em sua composição mecanismos que fazem com que os nutrientes adequados para as plantas sejam liberados de maneira compatível com suas demandas. Estes materiais são chamados de fertilizantes de liberação lenta. Uma revisão da literatura é realizada neste trabalho com o objetivo de avaliar as principais características dos fertilizantes de liberação lenta a base de materiais zeolíticos. As zeólitas são aluminosilicatos hidratados de metais alcalinos ou alcalinos terrosos e tem como principais aplicações o uso em tratamento de água e efluentes. As principais características das zeólitas são também abordadas buscando compreender suas propriedades potenciais para a aplicação proposta. Os benefícios em relação ao uso destes materiais na agricultura referem-se principalmente a sua notável seletividade catiônica e microporosidade. $\mathrm{O}$ uso de fertilizantes de liberação lenta a base de zeólitas se mostra uma possibilidade factível de utilização na agricultura, aumentando a eficiência e melhorando a sustentabilidade econômica, social e ambiental dos processos de produção agrícola.

Palavras-Chave: Fertilizantes, Liberação lenta, Zeólitas, Nutrientes, Produção sustentável.

\section{RESUMEN}

Los fertilizantes se agregan intencionalmente al suelo o a la superficie de la planta para proporcionar los nutrientes necesarios durante su vida y así aumentar la productividad. Los principales nutrientes

\footnotetext{
${ }^{1}$ Engenheiro Ambiental (Universidade do Extremo Sul Catarinense - UNESC), Doutor em Ciência e Engenharia de Materiais (Universidade Federal de Santa Catarina - UFSC), lisandrosimao@gmail.com;

${ }^{2}$ Engenheiro Têxtil (Universidade Estadual de Maringá - UEM), Doutor em Engenharia Química (UFSC), carlos.oliveira@ufsc.br;

${ }^{3}$ Curso de Engenharia Química, Instituto Latino-Americano de Tecnologia, Infraestrutura e Território (ILATIT), Universidade Federal da Integração Latino-Americana (UNILA), luis.lourenco@unila.edu.br;

${ }^{4}$ Engenheiro Agroindustrial-Agroquímico (Universidade Federal do Rio Grande - FURG), Estudante de mestrado em Engenharia Química (UFSC), afonso.silva@posgrad.ufsc.br;

${ }^{5}$ Engenheira de Alimentos (Universidade de Passo Fundo - UPF), Doutora em Engenharia Química

(UFSC), ellenfrodrigues@gmail.com;
} 


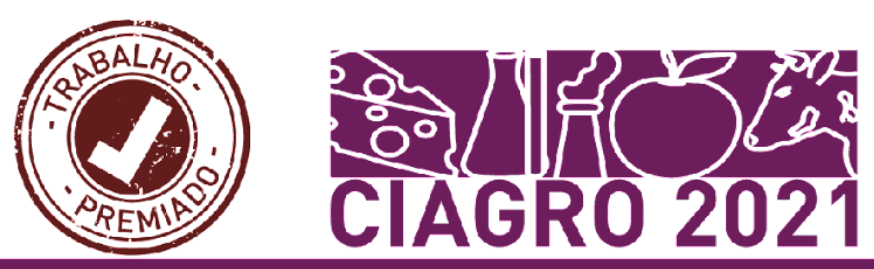

que se utilizan en la agricultura son el nitrógeno, el fósforo y el potasio, siendo el conjunto de estos macronutrientes el denominado N-P-K. El uso indiscriminado y prolongado de fertilizantes se ha convertido en una fuente importante de contaminación del suelo, el aire y el agua. El desequilibrio entre las tasas de liberación de nutrientes de los fertilizantes y las tasas de absorción por las plantas, hace que el proceso de fertilización del suelo sea menos eficiente. Para solucionar este problema, se pueden utilizar fertilizantes que tengan en su composición mecanismos que hagan que los nutrientes sean liberadas de forma compatible con las demandas de las plantas. Estos materiales se denominan fertilizantes de liberación lenta. En este trabajo se realiza una revisión de la literatura con el fin de evaluar las principales características de los fertilizantes de liberación lenta a base de materiales zeolíticos. Las zeolitas son aluminosilicatos hidratados de metales alcalinos o alcalinotérreos y tienen como aplicaciones principales el uso en el tratamiento de aguas y efluentes. Las principales características de las zeolitas también se abordan en un intento por comprender sus propiedades potenciales para la aplicación propuesta. Los beneficios en relación al uso de estos materiales en agricultura se refieren principalmente a su notable selectividad catiónica y microporosidad. El uso de fertilizantes de liberación lenta a base de zeolitas es una posibilidad factible de uso en agricultura, aumentando la eficiencia y mejorando la sostenibilidad económica, social y ambiental de los procesos productivos agrícolas.

Palabras Clave: Fertilizantes, Liberación lenta, Zeolitas, Nutrientes, Producción sustentable.

\begin{abstract}
Fertilizers are intentionally added to the soil or the surface of the plant to provide the necessary nutrients during its life and thus increase productivity. The main nutrients used in agriculture are nitrogen, phosphorus, and potassium, commonly called N-P-K. The indiscriminate and long-term use of fertilizers has become a relevant source of soil, air, and water pollution. The imbalance between the rates of release of nutrients from fertilizers and the absorption rates by plants makes the soil fertilization process less efficient. To solve this problem, you can use fertilizers that have in their composition mechanisms that make the nutrients suitable for the plant's demands. These materials are called slow-release fertilizers. A literature review is carried out in this work to evaluate the main characteristics of slow-release fertilizers based on zeolitic materials. Zeolites are hydrated aluminosilicates of alkaline or alkaline earth metals and have as main applications the use in water and effluent treatment. The main characteristics of zeolites are also addressed in an attempt to understand their potential properties for the proposed application. The benefits of the use of these materials in agriculture refer mainly to their remarkable cationic selectivity and microporosity. The use of slow-release fertilizers based on zeolites is a feasible possibility of use in agriculture, increasing efficiency and improving the economic, social, and environmental sustainability of agricultural production processes.
\end{abstract}

Keywords: Fertilizers, Slow release, Zeolites, Nutrients, Sustainable production. 


\section{FERTILIZANTES DE LIBERAÇÃO LENTA}

MATERIAIS ZEOLÍTICOS

USO DE MATERIAIS ZEOLÍTICOS COMO FERTILIZANTES

CONCLUSÕES

REFERÊNCIAS

\section{INTRODUÇÃO}

Com o crescimento da população ao longo dos anos, o setor agrícola está cada vez mais usando fertilizantes para aumentar a produtividade e consequente oferta de alimentos em todo o mundo (AZEEM et al., 2014). O uso excessivo de fertilizantes químicos na agricultura causa graves problemas ao meio ambiente pois apenas uma fração dos nutrientes é absorvida pelo solo e pelas plantas. O excesso de nutrientes presentes nos fertilizantes, tais como nitrogênio, fósforo e potássio, são normalmente lixiviados para os corpos d'água levando a eutrofização dos corpos hídricos. Em alguns casos, como do $\mathrm{N}$, pode-se ter perdas consideráveis pela volatilização para o ar (BANSIWAL et al., 2006; YUAN et al., 2017).

Sendo assim, há uma necessidade extrema de se mudar as práticas agronômicas, desenvolvendo fertilizantes amigáveis que facilitem a absorção máxima de nutrientes e consequentemente aumentem o rendimento das colheitas (LATEEF et al., 2016). Esta mudança de paradigma estaria coerente com as práticas de preservação ambiental, tornando o processo de produção de alimentos mais sustentável.

Neste cenário, a utilização de fertilizantes de liberação lenta tem sido estudada nos últimos anos para resolução dessa problemática econômica e ambiental do setor agrícola (LATEEF et al., 2016; PICKERING; MENZIES; HUNTER, 2002; SOUZA et al., 2018). Os fertilizantes de liberação lenta são materiais que em sua estrutura possuem mecanismos que retardam a liberação dos elementos nutricionais ao longo do tempo, melhorando a eficiência do fertilizante e da produção de alimentos, além de evitar a contaminação do solo, água e ar (YUAN et al., 2017).

As zeólitas são um grupo de minerais de ocorrência natural ou sintética que possuem estruturas cristalinas tridimensionais contendo em seu interior vazios e canais de tamanho molecular, sendo bastantes conhecidas por conta da sua alta capacidade de troca cationica (PICKERING; MENZIES; HUNTER, 2002) e utilização em diversos processos de remediação ambiental (LIU et al., 2016; VISA, 2016; ZHENG et al., 2019) e catálise (CHICA, 2013; HE et al., 2019; KHANDAN et al., 2015). 


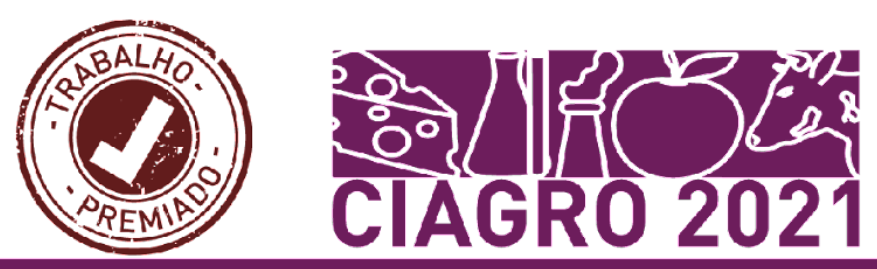

Muitos estudos tem sido realizados para aproveitar as características das zeólitas no desenvolvimento de fertilizantes com propriedades de liberação lenta (DWAIRI, 1998). Os benefícios em relação ao uso destes materiais na agricultura refere-se principalmente a sua notável seletividade catiônica e microporosidade (PARK et al., 2005), tornando-a ainda mais atrativa para a aplicação como fertilizantes. Em relação aos desafios, pode-se relatar a sua baixa capacidade de retenção de alguns nutrientes aniônicos, apesar de sua alta capacidade de troca iônica (BANSIWAL et al., 2006; PARK et al., 2005).

Neste trabalho, uma revisão da literatura é realizada para avaliar as principais características dos fertilizantes de liberação lenta a base de materiais zeolíticos. As principais características e propriedades das zeólitas são também abordadas buscando compreender suas propriedades potenciais para a aplicação proposta.

\section{FERTILIZANTES DE LIBERAÇÃO LENTA}

Os fertilizantes são um termo comum utilizado para os materiais que são intencionalmente adicionados no solo ou na superfície da planta com o objetivo de fornecer os nutrientes necessários no período de crescimento da cultura. São derivados de uma variedade de ingredientes naturais e materiais sintetizados, podendo estar na forma sólida ou líquida. Os fertilizantes comerciais usualmente utilizados na agricultura são nitrogênio $(\mathrm{N})$, fósforo $\left(\mathrm{P}_{2} \mathrm{O}_{5}\right)$ e potássio (K) (PURNOMO; SAPUTRA, 2021), sendo o conjuntos destes macronutrientes chamados de N-P-K. Estes fertilizantes podem ser adicionados no solo de maneira unitária utilizando apenas o nutriente de interesse, ou até mesmo de maneira conjunta, utilizando formulações mais complexas de fertilizantes contendo mais de um elemento na mistura (PURNOMO; SAPUTRA, 2021). A tabela 1 mostra os principais adubos simples utilizados para produção de outras formulações comerciais, sendo compostos principalmente pelos macronutrientes N-P-K.

Estes compostos nutricionais podem ser classificados de diferentes maneiras, podendo considerar o seu conteúdo nutricional, seu padrão de liberação e origem. Em relação a sua origem, podemos classificar os fertilizantes como orgânicos ou sintéticos. Os orgânicos são fabricados a partir de restos de organismos, enquanto que os sintéticos são fabricados a partir de gás natural ou minerais com processamentos específicos de acordo com o nutriente produzido (DIMKPA et al., 2020; PURNOMO; SAPUTRA, 2021).

Com o aumento das demandas por alimentos em todo o globo, é necessário o aumento 


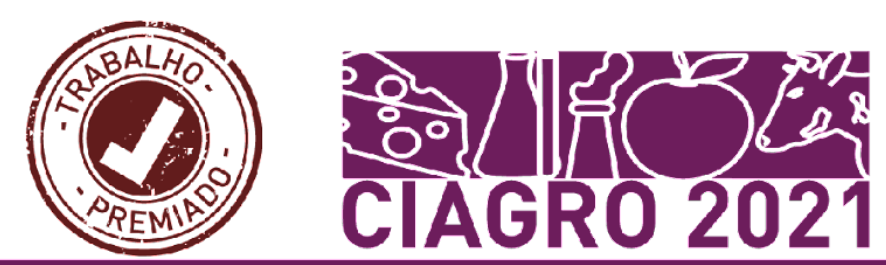

da produção agrícola para suprir as necessidades globais. Este aumento pode ser atingido minimizando as perdas por pragas e ervas daninhas ou utilizando agroquímicos e fertilizantes

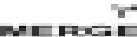

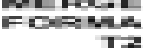
mais eficientes. Nesta linha de pensamento, os fertilizantes tem um papel importantíssimo no aumento da produção agrícola, mas aumentar o uso por si só pode não ser a melhor estratégia, já que durante a utilização há diversas perdas por processos como adsorção, degradação, escoamento e lixiviação. O desequilíbrio entre as taxas de liberação dos nutrientes oriundos dos fertilizantes e as taxas de absorção pelas raízes das plantas, tornam o processo de fertilização do solo menos eficientes, gerando custos para a produção de alimentos como um todo (BHARDWAJ et al., 2012; DIMKPA et al., 2020). Além disso, os autos índices de nutrientes desperdiçados aliado a sua alta solubilidade podem causar diversos problemas ao meio ambiente (BHARDWAJ et al., 2012; NOTARIO DEL PINO et al., 1995).

Tabela 01: Principais adubos simples utilizados em formulções comerciais

\begin{tabular}{ccc}
\hline Adubo & Nutriente & Teores minímos (\%) \\
Uréia & $\mathrm{N}$ & 44 \\
Nitrato de sódio & $\mathrm{N}$ & 15 \\
Nitrato de amônio & $\mathrm{N}$ & 32 \\
Sulfato de amônio & $\mathrm{N}$ & 20 \\
Nitrato de amônio e cálcio & $\mathrm{N}$ & 20 \\
& $\mathrm{Ca}$ & $2-8$ \\
& $\mathrm{Mg}$ & $1-5$ \\
Nitrato de cálcio & $\mathrm{N}$ & 14 \\
& $\mathrm{Ca}$ & $18-19$ \\
Superfosfato simples & $\mathrm{P}_{2} \mathrm{O}_{5}$ & 18 \\
& $\mathrm{~S}$ & $10-12$ \\
Superfosfato triplo & $\mathrm{P}_{2} \mathrm{O}_{5}$ & 41 \\
& $\mathrm{Ca}$ & $12-14$ \\
Monoamônio fosfato & $\mathrm{N}$ & 9 \\
& $\mathrm{P}_{2} \mathrm{O}_{5}$ & 48 \\
Diamônio fosfato & $\mathrm{N}$ & 16 \\
& $\mathrm{P}_{2} \mathrm{O}_{5}$ & 45 \\
Cloreto de potassio & $\mathrm{K}_{2} \mathrm{O}$ & 58 \\
Sulfato de potássio & $\mathrm{K}_{2} \mathrm{O}$ & 48 \\
Nitrato de potássio & $\mathrm{K}_{2} \mathrm{O}$ & 44 \\
& $\mathrm{~N}$ & 13 \\
\hline
\end{tabular}

Fonte: (BISSANI; MEURER; GIANELLO, 2021)

No caso do nitrogênio, um dos nutrientes mais importantes para o crescimento e desenvolvimento de diversas culturas agrícolas, seu uso está normalmente relacionado a grandes perdas após sua aplicação. Estas perdas resultam na maioria das vezes em impactos ao meio ambiente, tais como o aumento da produção de gases do efeito estufa devido a 


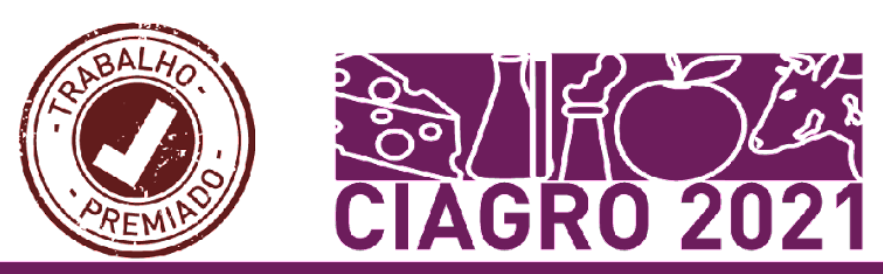

volatização de compostos como a amônia e óxido nitroso; e lixiviação de nitratos para o meio aquoso (DIMKPA et al., 2020). A contaminação das águas por nitratos é uma grande preocupação devido a sua toxicidade e efeitos nocivos no meio ambiente e saúde humana, tais como a síndrome do bebe azul, câncer, diabetes e danos ao fígado (BHARDWAJ et al., 2012). Para resolver este problema, pode-se usar fertilizantes que tem em sua composição mecanismos que fazem com que os nutrientes adequados para as plantas sejam liberados de maneira mais lenta e compatível com as demandas nutricionais das plantas (BHARDWAJ et al., 2012; LATEEF et al., 2016).

Alguns nutrientes são modificados quimicamente e estruturalmente buscando fornecer as plantas-alvo suprimentos constantes nos períodos mais necessários, ou seja, durante os picos de maior crescimento. Estes fertilizantes são comumente chamados de fertilizantes de liberação lenta (BHARDWAJ et al., 2012; LATEEF et al., 2016; PURNOMO; SAPUTRA, 2021).

Estes fertilizantes de liberação lenta possuem vantagens, tais como a capacidade de diminuir a taxa de perda, fornecendo nutrientes de forma sustentável, redução da frequência de aplicação e minimização dos potenciais efeitos negativos associados à sobre dosagem (PURNOMO; SAPUTRA, 2021) comentados anteriormente.

\section{MATERIAIS ZEOLÍTICOS}

As zeólitas são aluminosilicatos hidratados de metais alcalinos ou alcalinos terrosos (principalmente sódio, potássio, magnésio e cálcio) (LUZ, 1995; NOTARIO DEL PINO et al., 1995). Elas englobam variados minerais com características comuns e que podem ser naturais ou sintéticos. As zeólitas são normalmente estruturadas em redes cristalinas de tetraedros do tipo TO4, podendo o $\mathrm{T}$ ser $\mathrm{Si}, \mathrm{Al}, \mathrm{B}, \mathrm{Ge}, \mathrm{Fe}, \mathrm{P}, \mathrm{Co}$, dentre outros. Sua fórmula química é representada pela equação 1 , onde $\mathrm{M}$ é o cátion de valência $\mathrm{n}, \mathrm{m}$ é o número de moléculas de água e x+y é o número de tetraedros por célula unitária (LUZ, 1995).

$$
M_{\frac{x}{n}}\left[\left(\mathrm{AlO}_{2}\right)_{x}\left(\mathrm{SiO}_{2}\right)_{y}\right] \cdot \mathrm{mH}_{2} \mathrm{O} \text { (Equação 1) }
$$

A estrutura das zeólitas possui canais e cavidades interconectadas, onde encontra-se íons de compensação, moléculas de água ou outros sais (LUZ, 1995; NOTARIO DEL PINO 


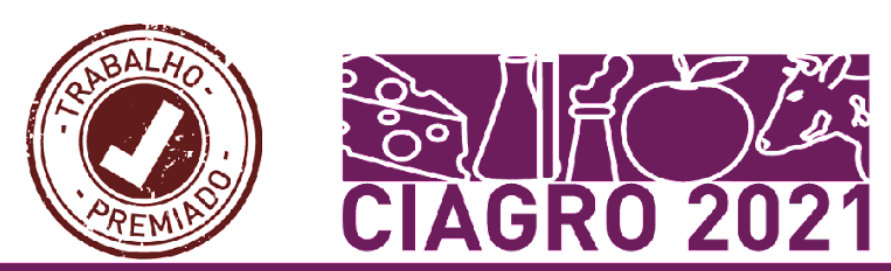

et al., 1995). Estas características de microporosidade fazem com que estes materiais possuam uma grande área de superfície específica interna quando comparado a sua superfície externa. Psere

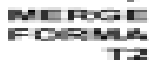
A porosidade desses materiais permitem ainda a transferência de matéria, limitado pelo tamanho dos poros (LUZ, 1995).

Estes materiais possuem como principais propriedades físico-químicas a superfície específica; volume de vazios; índice de refração; massa específica; capacidade de adsorção de gás (YAN et al., 2019), capacidade de troca catiônica; alto grau de hidratação; baixa densidade; condutividade elétrica; e propriedades catalíticas (CHICA, 2013; LUZ, 1995).

Os principais usos das zeólitas (Figura 1) são na construção civil: como blocos de rocha, pozolanas e agregados leves (LUZ, 1995; RAMEZANIANPOUR et al., 2015; TRAN et al., 2019); tratamento de água e efluentes (LIU et al., 2016); catálise (HU et al., 2021); Aplicações biomédicas (SERATI-NOURI et al., 2020), agentes microbianos (DUTTA; WANG, 2019), tratamento de resíduos radioativos (JIMÉNEZ-REYES; ALMAZÁN-SÁNCHEZ; SOLACHE-RÍOS, 2021), captura de $\mathrm{CO}_{2}$ (KUMAR; SRIVASTAVA; KOH, 2020), carga na indústria de papel; detergentes, nutrição animal e na agricultura (LUZ, 1995).

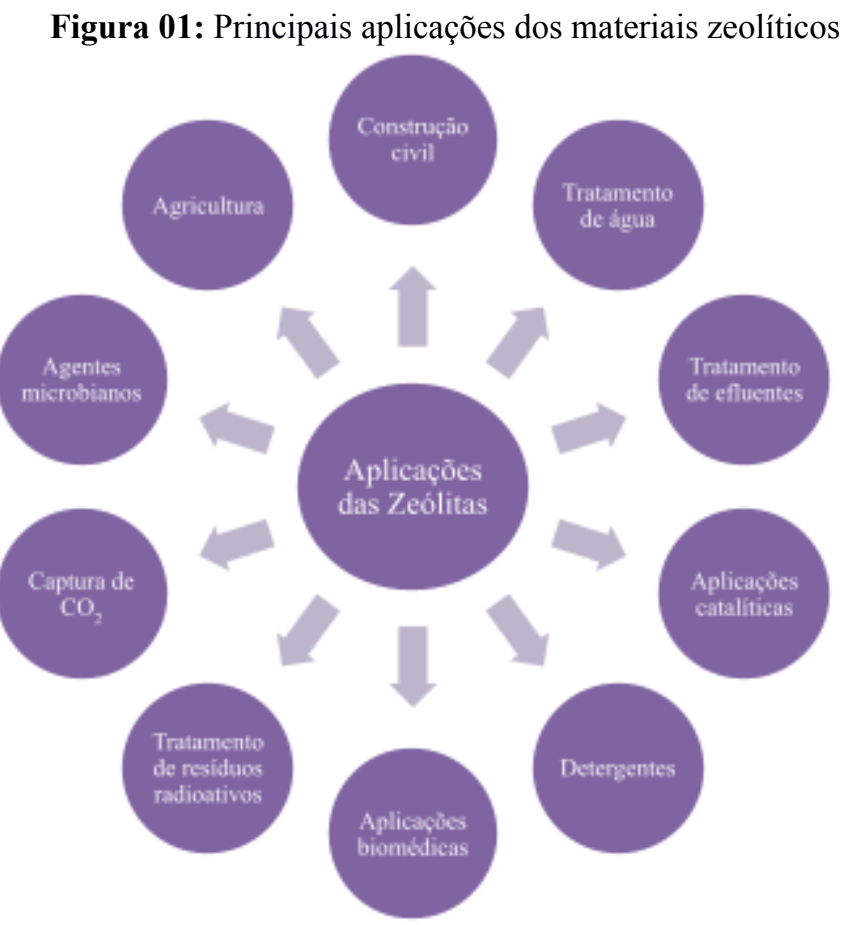

Fonte: Dos autores (2021).

$\mathrm{Na}$ agricultura, o Japão vem usando a clinoptilolita no melhoramento da qualidade do solo aproveitando a capacidade de troca catiônica desses materiais e favorecendo a retenção 


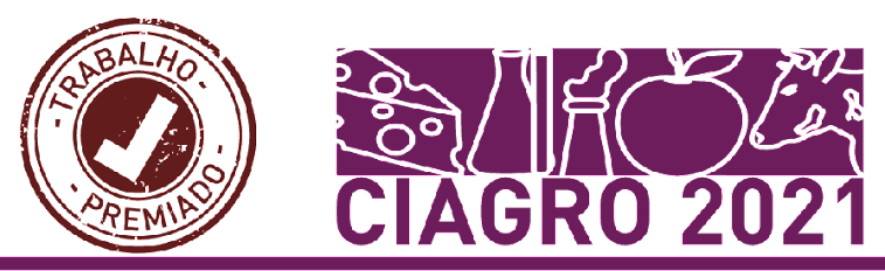

de nutrientes pelo solo, tais como potássio e nitrogênio. Sua alta capacidade de troca catiônica também faz das zeólitas bons suportes para herbicidas e fungicidas (LUZ, 1995). O uso de zeólitas na agricultura pode ter outras funções importantes, tais como boa capacidade de retenção de água, aumento da disponibilidade de nutrientes e melhora das propriedades físicas, químicas e biológicas do solo (KHAN et al., 2021).

Recentemente, estudos tem avaliado a sua utilização como fertilizantes de liberação lenta, assunto debatido com profundidade no tópico subsequente. Suas propriedades de alta área de superfície específica, capacidade de troca catiônica e microporosidade são algumas das motivações dos diversos estudos presentes na literatura.

\section{USO DE MATERIAIS ZEOLÍTICOS COMO FERTILIZANTES}

Neste tópico uma revisão da literatura sobre os principais trabalhos de fertilizantes de liberação lenta oriundos de materiais zeolíticos (Quadro 01) são relatados buscando compreender as potencialidades, desafios e principais vantagens do uso das zeólitas na agricultura.

O uso indiscriminado e de longo prazo de fertilizantes fosfatados tem se tornado uma fonte relevante de poluição do solo e água. A recuperação do fósforo em recursos hídricos pode, além de resolver o problema da eutrofização, gerar oportunidade de se obter novas fontes secundárias deste fertilizante. Da mesma forma, a liberação lenta de potássio em zeólitas, quando comparado aos fertilizantes tradicionais, evitam as perdas por lixiviação, além de permitir a retenção de outros nutrientes (principalmente nitrogênio) em sua estrutura porosa. Estes fatores aumentam a absorção das plantas e consequentemente aumentam a produtividade do solo, trazendo benefícios tanto ambientais quanto econômicos para o processo, já que reduzem as taxas de aplicação de fertilizantes químicos no solo. Neste sentido, a síntese de zeólitas de K contendo outros nutrientes essenciais (tais como fósforo) se torna um interesse chave para o agronegócio (HERMASSI et al., 2020). Nesta linha de pensamento, Hermassi et al. (2020) investigou a sorção de fosfato por K-zeólitas sintetizadas a partir de cinzas volantes por conversão hidrotérmica. Os resultados obtidos mostraram que as zeólitas sintetizadas são materiais promissores para a recuperação de íons de $\mathrm{P}$ de efluentes sanitários. Por conseguinte, as zeólitas contendo fósforo se tornam um potencial material fertilizante para ser utilizado na recuperação de áreas degradadas (HERMASSI et al., 2020). 


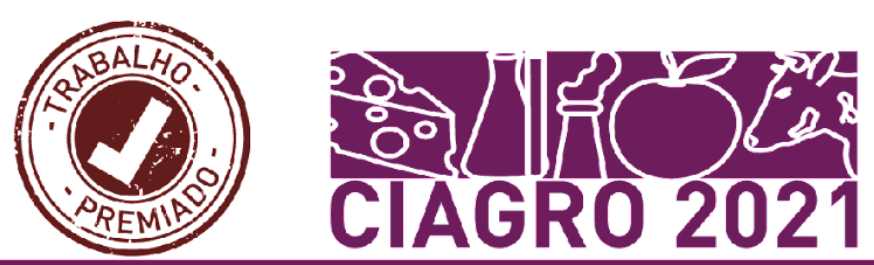

O uso de zeólitas $\mathrm{F}$ pode fornecer além do $\mathrm{K}$ ao solo, outras funções importantes, tais como correção de acidez, retenção de água e de outros nutrientes. Yuan et al. (2017) investigou a influência da concentração de $\mathrm{KOH}$ e do tempo de reação na síntese de zeólitas $\mathrm{F}$ a partir de feldspatos. Os resultados de DRX indicaram a presença de zeólita K-G $\left(\mathrm{K}_{2} \mathrm{Al}_{2} \mathrm{SiO}_{6} \cdot \mathrm{H}_{2} \mathrm{O}\right)$ com estrutura hexagonal quando utilizado concentrações de $2 \mathrm{~mol} / \mathrm{kg}$ de $\mathrm{KOH}$; e de zeólitas $\mathrm{F}\left(\mathrm{KAlSiO}_{4} \cdot 1,5 \mathrm{H}_{2} \mathrm{O}\right)$ com estrutura tetragonal quando utilizados concentrações de 4-5 mol $/ \mathrm{kg}$. Os resultados de liberação de nutrientes, demonstram que após 42 d houve uma liberação de $68,97 \%$ do potássio presente nas zeólitas formadas (YUAN et al., 2017).

Li et al. (2014) realizou uma síntese de zeólitas merlinoita usando cinzas volantes e $\mathrm{KOH}$ para uso como fertilizantes de liberação lenta em minas degradadas e plantações com alta demanda de nutrientes, como na cultura do girassol. Além dos benefícios da aplicação do material obtido, a valorização de um resíduo no processo, aumentou seu valor econômico e reduziu os custos ambientais e econômicos do envio destes resíduos para aterros. Os resultados obtidos mostraram que zeólitas merlinoita de alta qualidade (CEC de $160 \mathrm{cmol} / \mathrm{kg}$ ) foi produzida utilizando soluções de $\mathrm{KOH}$ de $5 \mathrm{M}$, temperatura de ativação de $150{ }^{\circ} \mathrm{C}$, tempo de ativação de $8 \mathrm{~h}$ e razão $\mathrm{KOH} /$ cinza volante de $2 \mathrm{~L} / \mathrm{kg}$. Além disso, as conclusões dos autores demonstraram que as zeólitas produzidas são fertilizantes de K com liberação lenta eficientes, podendo ser utilizado em plantações que requerem alto teor de reposição nutricional e solos pobres de áreas degradadas (LI et al., 2014).

Quadro 01: Principais zeólitas utilizadas como fertilizantes de liberação lenta.

\begin{tabular}{|c|c|c|}
\hline Zeólita utilizada & Nutrientes de interesse & Referência \\
\hline K-Zeólitas & $\mathrm{K} \mathrm{e} \mathrm{P}$ & Hermassi et al. (2020) \\
\hline Zeólita F & $\mathrm{K}$ & Yuan et al. (2017) \\
\hline zeólita K-G & $\mathrm{K}$ & Yuan et al. (2017) \\
\hline Merlinoita & $\mathrm{K}$ & Li et al. (2014) \\
\hline Filipsita & $\mathrm{K} \mathrm{e} \mathrm{P}$ & Notario Del Pino et al. (1995) \\
\hline Clinoptilolita & $\mathrm{N}$ & Souza et al. (2018) \\
\hline zeólitas Na-P & $\mathrm{N}$ & Park et al. (2005) \\
\hline Clinoptilolita & $\mathrm{N}$ & Bhardwaj et al. (2012) \\
\hline Zeólita A & $\mathrm{P}$ & Bansiwal et al. (2006) \\
\hline Clinoptilolita & $\mathrm{P}, \mathrm{N} \mathrm{e} \mathrm{K}$ & Pickering et al. (2002) \\
\hline
\end{tabular}

Fonte: Elaborado pelos autores a partir de várias fontes (2021).

Notario Del Pino et al. (1995) fez um estudo comparativo de liberação de P e K utilizando fertilizantes a base de zeólitas e $\mathrm{KH}_{2} \mathrm{PO}_{4}$ (fertilizante comercial) em um reator de percolação com fluxo de solução constante. A zeólita Filipsita de Tenerife foi utilizada como 


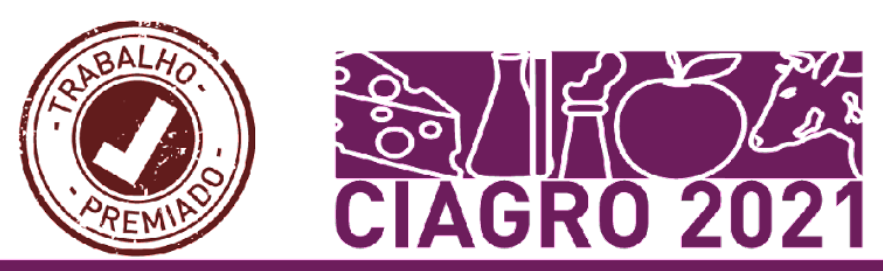

matriz para preparar os fertilizantes de $\mathrm{P}$ e K com liberação controlada. Os resultados mostraram que os fertilizantes a base de zeólitas fornecem $\mathrm{P}$ ao sistema após mais de 70 dias de percolação contínua, enquanto que o $\mathrm{P}$ do $\mathrm{KH}_{2} \mathrm{PO}_{4}$ se esgota após 50 dias. As conclusões dos autores foram que os fertilizantes de zeólitas filipsita se mostraram promissores para liberação controlada de potássio (NOTARIO DEL PINO et al., 1995).

O nitrogênio é um nutriente essencial e limitante para o crescimento das plantas, principalmente para a cultura do arroz. A ureia, fertilizante comumente utilizado para fornecimento de $\mathrm{N}$, é altamente solúvel e rapidamente convertida em amônio $\left(\mathrm{NH}_{4}^{+}\right)$, o qual pode ser perdido por escoamento, lixiviação e volatização (AZEEM et al., 2014; MAGHSOODI et al., 2020; SOUZA et al., 2018). Os métodos atuais de fertilização dos solos reduzem a eficiência do uso do nitrogênio pelas plantas, limitando seu crescimento e contribuindo para a poluição ambiental no que se refere as emissões de gases perigosos e eutrofização dos recursos hídricos (AZEEM et al., 2014). Uma alternativa para reduzir as perdas de nitrogênio é desenvolver os fertilizantes de liberação lenta, onde a ureia é revestida por espécies inertes e biodegradáveis, criando uma barreira física ao redor dos grânulos e controlando a liberação do nitrogênio pela difusão da ureia pelo carreador, aumentando o tempo de liberação de nutrientes quando comparado a ureia tradicional (AZEEM et al., 2014; COSTA et al., 2013; SOUZA et al., 2018).

Nesse sentido, Maghsoodi (2020) investigou a liberação de nitrogênio (N) utilizando diferentes matrizes de fertilizantes, tais como ureia biochar, hidrocarburante, zeólitas e nanohíbridos de ureia-hidroxiapatita. Os resultados mostraram que as zeólitas são fertilizantes de liberação lenta eficientes, reduzindo a liberação de ureia em 9 vezes quando comparado ao padrão utilizado (MAGHSOODI et al., 2020). Souza et al. (2018) desenvolveu um fertilizante a base de ureia e zeólita clinoptilolita em comprimidos para uso como fertilizantes de liberação lenta. A possibilidade de interação com íons amônia, aliado a alta troca catiônica das zeólitas clinoptilolita foram as motivações para desenvolver o estudo. Os resultados mostraram que não houve interações químicas entre os componentes da formulação, mas as formulações foram capazes de diminuir em até 53\% a quantidade de amônia volatilizada (SOUZA et al., 2018).

Os estudos sobre zeólitas como fertilizantes de liberação lenta são normalmente limitados aos nutrientes em formas catiônicas, a citar, $\mathrm{NH}_{4}^{+}$e $\mathrm{K}^{+}$. No caso de nutrientes aniônicos, tais como $\mathrm{NO}_{3}{ }^{-}$e $\mathrm{PO}_{4}{ }^{3-}$ o carregamento em materiais zeoliticos não modificados são insignificantes. Estudos e pesquisas que deixem estes materiais com afinidades por ânions se tornam importantes para uso como fertilizantes de liberação lenta (BANSIWAL et al., 2006; 


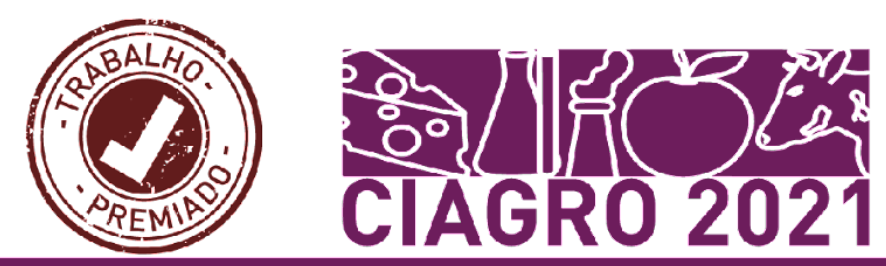

BHARDWAJ et al., 2012).

Alternativas para aumentar a proporção dos nutrientes nas zeólitas tem sido estudada, como por exemplo, a formação de sais estáveis nos poros da zeólita (oclusão de sal). Esta técnica pode inclusive exibir propriedades de liberação lenta dos nutrientes. Park et al. (2005) avaliou zeólitas Na-P ocluídas por sais de $\mathrm{NH}_{4} \mathrm{NO}_{3}(\mathrm{~N}-\mathrm{NaP})$ usando uma solução simulada de solo e água deionizada como soluções de lixiviação. A solução de solo afetou a liberação de $\mathrm{NH}_{4}^{+}$e $\mathrm{NO}_{3}^{-}$diferentemente, enquanto a água deionizada liberou quase os mesmos equivalentes desses íons. Isso indica claramente que a liberação de íons do zeólita ocluído por sal envolve duas reações diferentes, troca catiônica e dissolução. Os resultados apresentados indicam que as zeólitas ocluídos por $\mathrm{NH}_{4} \mathrm{NO}_{3}$ podem ser utilizadas como fertilizantes de liberação lenta e aumentar a sua eficiência de fertilização quando utilizadas no solo (PARK et al., 2005).

As propriedades aniônicas podem ser transmitidas para superfície zeolítica usando surfactantes. Bhardwaj et al. (2012) sintetizou zeólitas clinoptilolita e modificou sua estrutura utilizando o surfactante brometo de hexadeciltrimetilamônio (HDTMAB) e brometo de dioctadecildimetilamônio (DODMAB). Os produtos obtidos foram primeiramente utilizados para remoção de nitratos e posteriormente para uso como fertilizantes de liberação lenta. Os resultados mostraram uma capacidade de adsorção muito maior do que os materiais não modificados: $30,58 \mathrm{mg} / \mathrm{g}$ para clinoptilolita sem carregamento, $75,19 \mathrm{mg} / \mathrm{g}$ para clinoptilolita carregada com DODMAB e 125,00 mg/g para clinoptilolita carregada com HDTMAB. Por fim, os testes de liberação lenta mostraram que ainda há uma liberação de $\mathrm{NO}_{3}{ }^{-}$após 15-20 dias nos estudos de lixiviação (BHARDWAJ et al., 2012).

Bansiwal et al. (2006) investigou a viabilidade do uso de zeólitas modificadas com surfactante como fertilizantes de liberação lenta de P. A zeólita A foi modificada usando brometo de hexadeciltrimetilamónio, um tensoativo catiônico que aumenta a capacidade de reter ânions fosfatos. Os resultados mostraram que o P presente no surfactante ficou disponível após $1080 \mathrm{~h}$ de percolação contínua, enquanto que o $\mathrm{P}$ provindo do $\mathrm{KH}_{2} \mathrm{PO}_{4}$ ficou disponível até 264 h. Por fim, pôde-se concluir que o produto obtido é um bom sorvente para íons fosfatos, tendo uma liberação lenta de P durantes as condições testadas (BANSIWAL et al., 2006).

Neste estudo, Pickering et al. (2002), avaliou o uso de zeólitas clinoptilolita juntamente com rochas fosfáticas com objetivo de aumentar a absorção de P por girassóis. A combinação das zeólita e da rocha fosfática atuou como um fertilizante de troca, trocando $\mathrm{Ca}^{2+}$ nas zeólitas em resposta à absorção de nutrientes catiônicos pela planta $\left(\mathrm{NH}_{4}^{+}\right.$ou $\left.\mathrm{K}^{+}\right)$, 
aumentando assim a dissolução da rocha fosfática (PICKERING; MENZIES; HUNTER, 2002).

\section{CONCLUSÕES}

O aumento da população mundial e consequentemente, o aumento do consumo de alimentos faz com que as técnicas agrícolas se atualizem para suprir as demandas globais em relação a produção de alimentos.

O uso excessivo de fertilizantes tradicionais, tornam-se ineficazes para suprir estas demandas, já que as taxas de absorção das plantas não consomem as quantidades utilizadas, trazendo desperdícios e principalmente impactos ambientais ao solo, ar e água.

O uso de fertilizantes de liberação lenta a base de zeólitas se mostram uma possibilidade factível de utilização na agricultura com os objetivos de aumentar a eficiência e melhorar a sustentabilidade econômica, social e ambiental dos processos de produção agrícola.

\section{REFERÊNCIAS}

AZEEM, B.; KUSHAARI, K.; MAN, Z. B.; BASIT, A.; THANH, T. H. Review on materials \&amp; methods to produce controlled release coated urea fertilizer. Journal of Controlled Release, v. 181, p. 11-21, maio 2014.

BANSIWAL, A. K.; RAYALU, S. S.; LABHASETWAR, N. K.; JUWARKAR, A. A.; DEVOTTA, S. Surfactant-Modified Zeolite as a Slow Release Fertilizer for Phosphorus. Journal of Agricultural and Food Chemistry, v. 54, n. 13, p. 4773-4779, jun. 2006.

BHARDWAJ, D.; SHARMA, M.; SHARMA, P.; TOMAR, R. Synthesis and surfactant modification of clinoptilolite and montmorillonite for the removal of nitrate and preparation of slow release nitrogen fertilizer. Journal of Hazardous Materials, v. 227-228, p. 292-300, ago. 2012.

BISSANI, C. A.; MEURER, E. J.; GIANELLO, C. Fertilizantes e Formulações

Comerciais. Disponível em: $<$ https://www.ufrgs.br/agronomia/materiais/[000456916] p. 239-2490001.pdf>.

CHICA, A. Zeolites: Promised Materials for the Sustainable Production of Hydrogen. ISRN Chemical Engineering, v. 2013, p. 1-19, 31 jan. 2013.

COSTA, M. M. E.; CABRAL-ALBUQUERQUE, E. C. M.; ALVES, T. L. M.; PINTO, J. C.; FIALHO, R. L. Use of Polyhydroxybutyrate and Ethyl Cellulose for Coating of Urea 


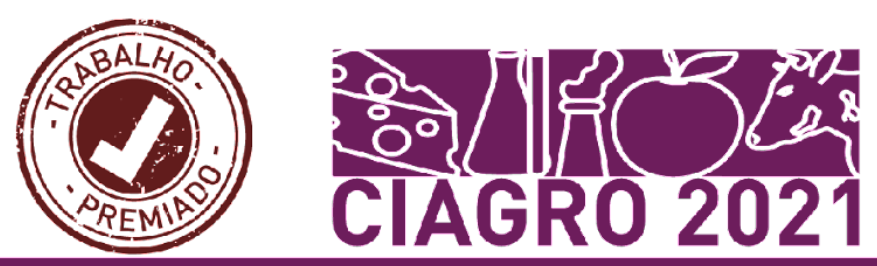

Granules. Journal of Agricultural and Food Chemistry, v. 61, n. 42, p. 9984-9991, 23 out. 2013.

DIMKPA, C. O.; FUGICE, J.; SINGH, U.; LEWIS, T. D. Development of fertilizers for enhanced nitrogen use efficiency - Trends and perspectives. Science of The Total Environment, v. 731, p. 139113, ago. 2020.

DUTTA, P.; WANG, B. Zeolite-supported silver as antimicrobial agents. Coordination Chemistry Reviews, v. 383, p. 1-29, mar. 2019.

DWAIRI, I. M. Evaluation of Jordanian zeolite tuff as a controlled slow-release fertilizer for NH 4 +. Environmental Geology, v. 34, n. 1, p. 1-4, 14 abr. 1998.

HE, P. Y.; ZHANG, Y. J.; CHEN, H.; HAN, Z. C.; LIU, L. C. Low-energy synthesis of kaliophilite catalyst from circulating fluidized bed fly ash for biodiesel production. Fuel, v. 257, p. 116041, dez. 2019.

HERMASSI, M.; VALDERRAMA, C.; FONT, O.; MORENO, N.; QUEROL, X.; BATIS, N. H.; CORTINA, J. L. Phosphate recovery from aqueous solution by K-zeolite synthesized from fly ash for subsequent valorisation as slow release fertilizer. Science of The Total

Environment, v. 731, p. 139002, ago. 2020.

HU, G.; YANG, J.; DUAN, X.; FARNOOD, R.; YANG, C.; YANG, J.; LIU, W.; LIU, Q. Recent developments and challenges in zeolite-based composite photocatalysts for environmental applications. Chemical Engineering Journal, v. 417, p. 129209, ago. 2021.

JIMÉNEZ-REYES, M.; ALMAZÁN-SÁNCHEZ, P. T.; SOLACHE-RÍOS, M. Radioactive waste treatments by using zeolites. A short review. Journal of Environmental Radioactivity, v. 233, p. 106610, jul. 2021.

KHAN, M. Z. H.; ISLAM, M. R.; NAHAR, N.; AL-MAMUN, M. R.; KHAN, M. A. S.; MATIN, M. A. Synthesis and characterization of nanozeolite based composite fertilizer for sustainable release and use efficiency of nutrients. Heliyon, v. 7, n. 1, p. e06091, jan. 2021.

KHANDAN, N.; ZIARATI, MAHMOUD; KARKEABADI, R.; GHAFOURI ROOZBAHANI, M. A. Hydrogen production via steam reforming of LPG on Ni/Zeolite catalysts. Iranian Journal of Hydrogen \& Fuel Cell, v. 1, n. 4, p. 233-238, 2015.

KUMAR, S.; SRIVASTAVA, R.; KOH, J. Utilization of zeolites as $\mathrm{CO} 2$ capturing agents: Advances and future perspectives. Journal of CO2 Utilization, v. 41, p. 101251, out. 2020.

LATEEF, A.; NAZIR, R.; JAMIL, N.; ALAM, S.; SHAH, R.; KHAN, M. N.; SALEEM, M. Synthesis and characterization of zeolite based nano-composite: An environment friendly slow release fertilizer. Microporous and Mesoporous Materials, v. 232, p. 174-183, set. 2016.

LI, J.; ZHUANG, X.; FONT, O.; MORENO, N.; VALLEJO, V. R.; QUEROL, X.; TOBIAS, A. Synthesis of merlinoite from Chinese coal fly ashes and its potential utilization as slow release K-fertilizer. Journal of Hazardous Materials, v. 265, p. 242-252, jan. 2014. 
LIU, Y.; YAN, C.; ZHANG, Z.; WANG, H.; ZHOU, S.; ZHOU, W. A comparative study on fly ash, geopolymer and faujasite block for $\mathrm{Pb}$ removal from aqueous solution. Fuel, v. 185, p. $181-189,2016$.

LUZ, A. B. DA. Zeólitas: propriedades e usos industriais. Rio de Janeiro: CETEM/CNPq, 1995.

MAGHSOODI, M. R.; NAJAFI, N.; REYHANITABAR, A.; OUSTAN, S. Hydroxyapatite nanorods, hydrochar, biochar, and zeolite for controlled-release urea fertilizers. Geoderma, v. 379, p. 114644, dez. 2020.

NOTARIO DEL PINO, J. S.; ARTEAGA PADRÓN, I. J.; GONZÁLEZ MARTIN, M. M.; GARCIA HERNÁNDEZ, J. E. Phosphorus and potassium release from phillipsite-based slow-release fertilizers. Journal of Controlled Release, v. 34, n. 1, p. 25-29, abr. 1995.

PARK, M.; KIM, J. S.; CHOI, C. L.; KIM, J.-E.; HEO, N. H.; KOMARNENI, S.; CHOI, J. Characteristics of nitrogen release from synthetic zeolite Na-P1 occluding NH4NO3. Journal of Controlled Release, v. 106, n. 1-2, p. 44-50, ago. 2005.

PICKERING, H. W.; MENZIES, N. W.; HUNTER, M. N. Zeolite/rock phosphate-a novel slow release phosphorus fertiliser for potted plant production. Scientia Horticulturae, v. 94, n. 3-4, p. 333-343, jun. 2002.

PURNOMO, C. W.; SAPUTRA, H. Manufacturing of slow and controlled release fertilizer. In: Controlled Release Fertilizers for Sustainable Agriculture. [s.1.] Elsevier, 2021.p. 95-110.

RAMEZANIANPOUR, A. A.; MOUSAVI, R.; KALHORI, M.; SOBHANI, J.; NAJIMI, M. Micro and macro level properties of natural zeolite contained concretes. Construction and Building Materials, v. 101, p. 347-358, dez. 2015.

SERATI-NOURI, H.; JAFARI, A.; ROSHANGAR, L.; DADASHPOUR, M.; PILEHVAR-SOLTANAHMADI, Y.; ZARGHAMI, N. Biomedical applications of zeolite-based materials: A review. Materials Science and Engineering: C, v. 116, p. 111225, nov. 2020.

SOUZA, I. M. S.; GURGEL, G. C. S.; MEDEIROS, A. M.; ZONTA, E.; RUIZ, J. A. C.; PASKOCIMAS, C. A.; MOTTA, F. V.; BOMIO, M. R. D. The use of clinoptilolite as carrier of nitrogened fertilizer with controlled release. Journal of Environmental Chemical Engineering, v. 6, n. 4, p. 4171-4177, ago. 2018.

TRAN, Y. T.; LEE, J.; KUMAR, P.; KIM, K.-H.; LEE, S. S. Natural zeolite and its application in concrete composite production. Composites Part B: Engineering, v. 165, p. 354-364, maio 2019.

VISA, M. Synthesis and characterization of new zeolite materials obtained from fly ash for heavy metals removal in advanced wastewater treatment. Powder Technology, v. 294, p. 338-347, jun. 2016.

YAN, B.; YU, S.; ZENG, C.; YU, L.; WANG, C.; ZHANG, L. Binderless zeolite NaX 


\section{microspheres with enhanced $\mathrm{CO} 2$ adsorption selectivity. Microporous and Mesoporous}

Materials, v. 278, p. 267-274, abr. 2019.

YUAN, J.; YANG, J.; MA, H.; CHANG, Q. Preparation of Zeolite F as Slow Release

Fertilizers from K-Feldspar Powder. ChemistrySelect, v. 2, n. 33, p. 10722-10726, 21 nov. 2017.

ZHENG, Z.; MA, X.; ZHANG, Z.; LI, Y. In-situ transition of amorphous gels to Na-P1 zeolite in geopolymer: Mechanical and adsorption properties. Construction and Building Materials, v. 202, p. 851-860, mar. 2019. 